
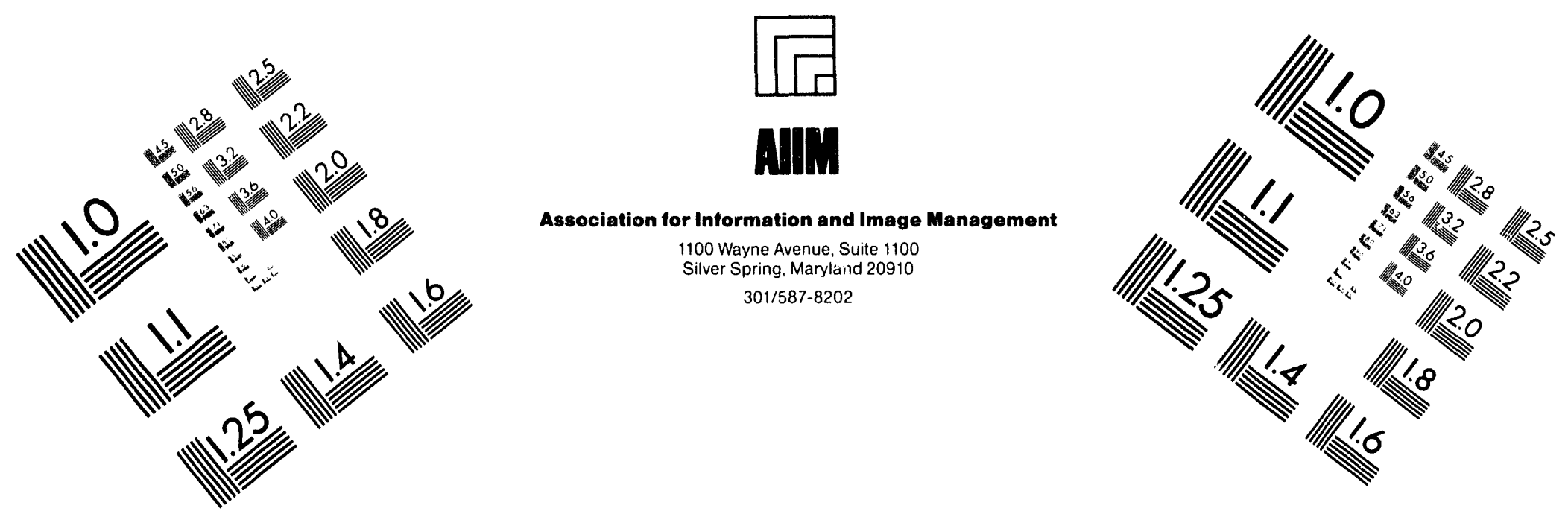

Centimeter

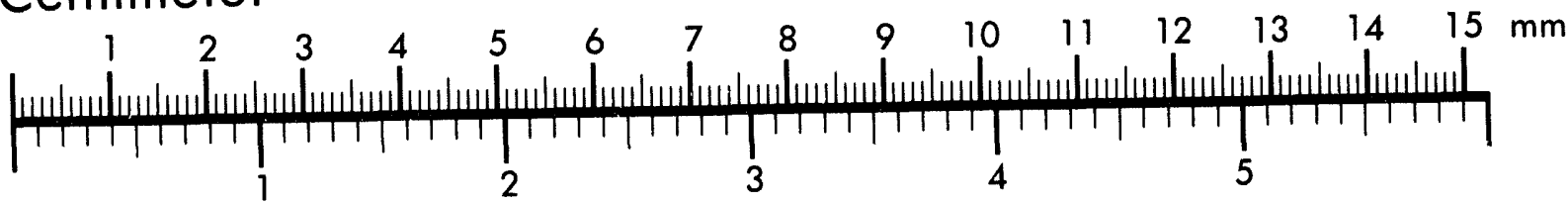
Inches
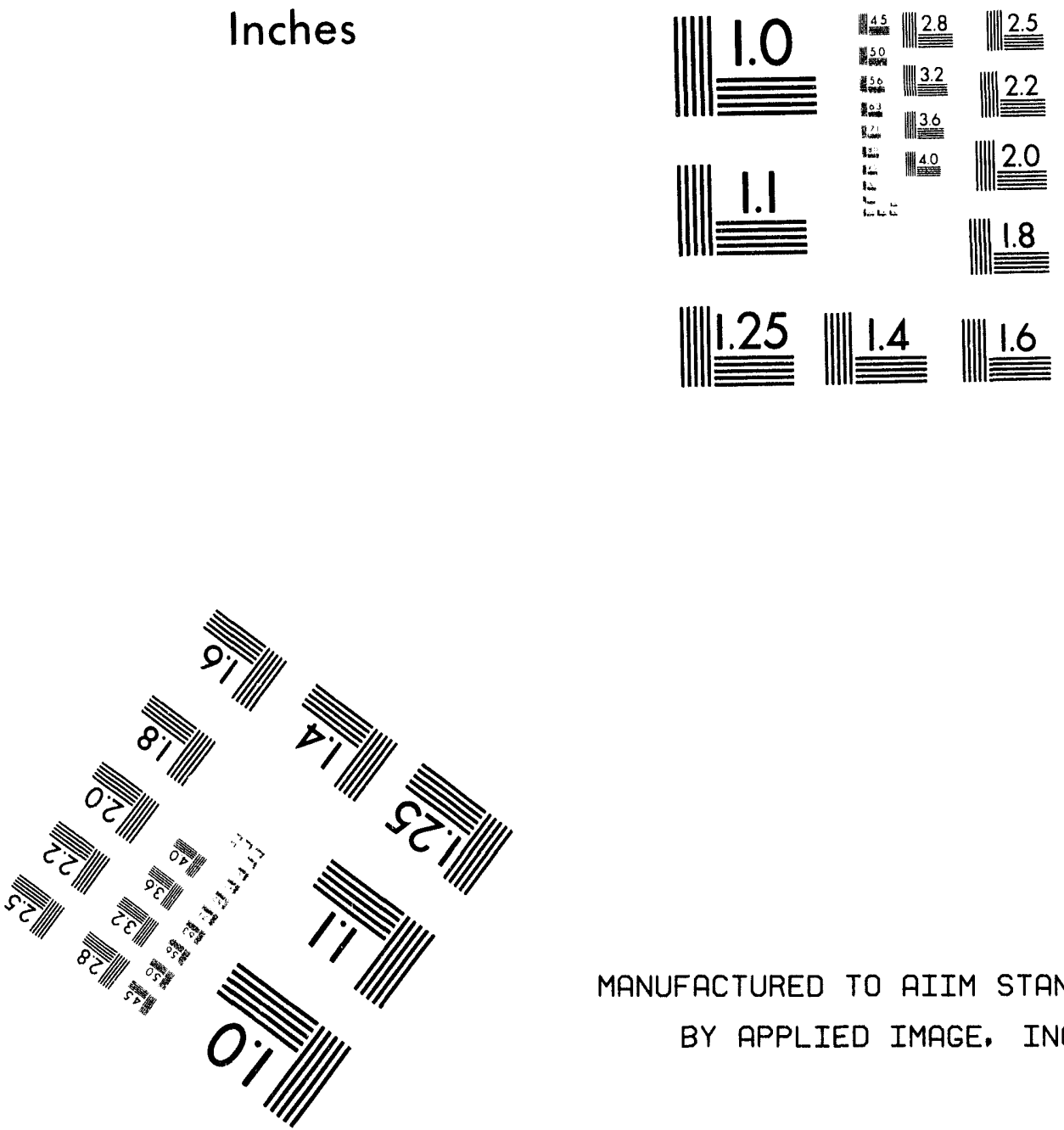

MANUFACTURED TO AIIM STANDARDS

BY APPLIED IMAGE, INC.

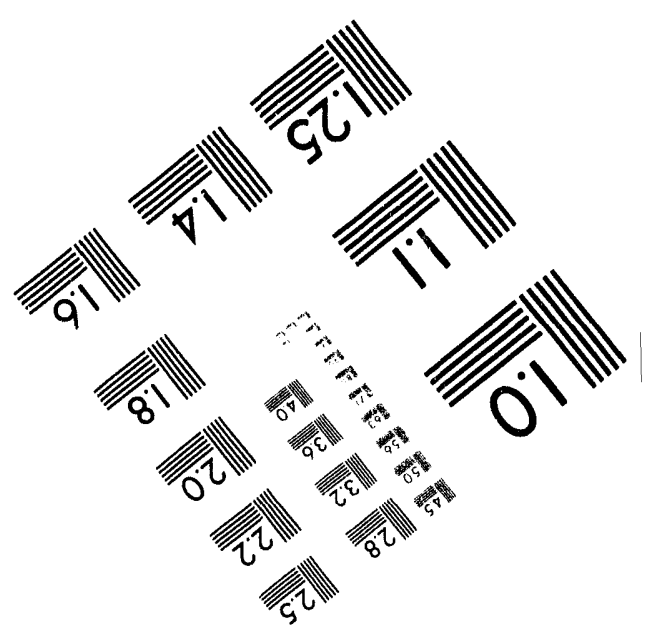




$$
\overrightarrow{\mathrm{o}}
$$




\section{La-ur- $94-1835$}

Title:

TONGUES, BOTTLES, AND DISCONNECTED LOOPS: THE OPENING AND CLOSING OF THE INTERPLANETARY MAGNETIC FIELD

Author(s):

David J. McComas

Submitted to:

U.S. National Report to the IUGG (1991-1994)

\section{Los Alamos}

NATIONAL LABORATORY

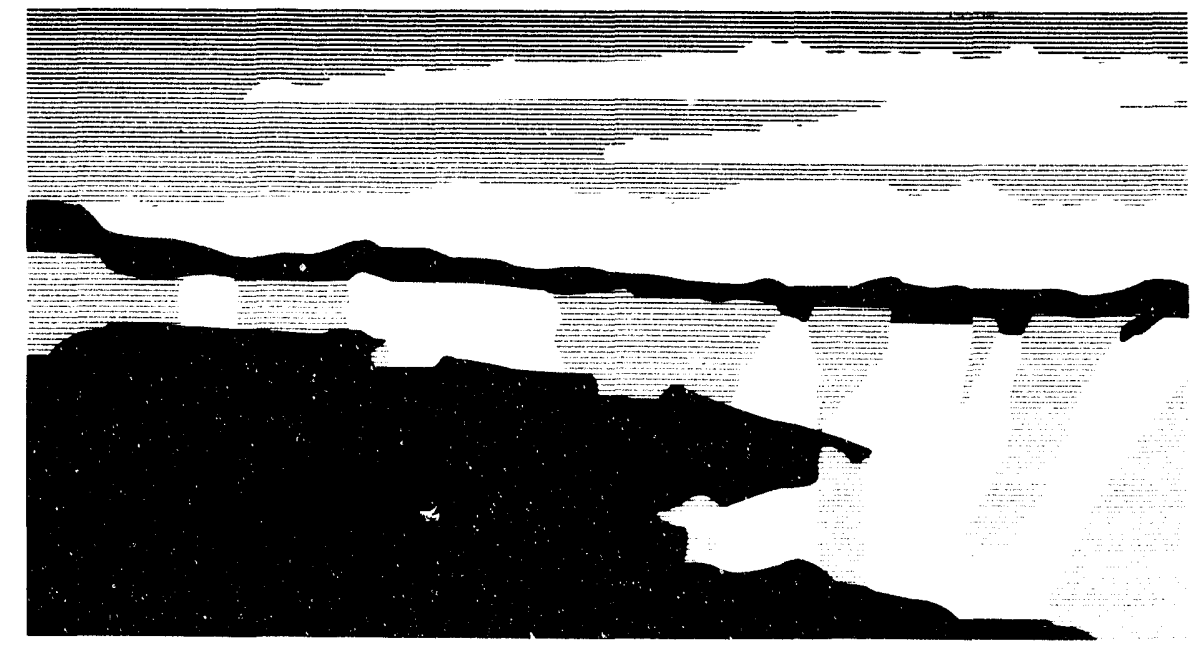

Los Alamos National Laboratory, an affirmative action/equal opportunity employer, is operated by the University of California for the U.S. Department of Energy under contract W-7405-ENG-36. By acceptance of this article, the publisher recognizes that the U.S. Government retains a nonexclusive, royalty-free license to publish or reproduce the published form of this contribution, or to allow others to do so, for U.S. Government purposes. The Los Alamos National Laboratory requests that the publisher identify this article as work performed under the auspices of the U.S. Department of Energy.

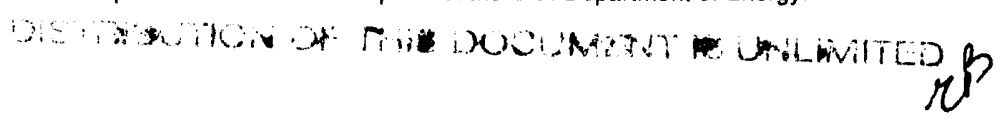




\title{
Tongues, Bottles, and Disconnected Loops: The Opening and Closing of the Interplanetary Magnetic Field
}

\author{
David J. McComas \\ Space and Atmospheric Sciences Group \\ Los Alamos National Laboratory, Los Alamos, NM 87545 USA
}

\begin{abstract}
For years the field of Space Physics has had a problem, a really big problem for it occurs on the largest spatial scales in Space Physics - across the entire region under the Sun's influence, the heliosphere. The problem is that the Sun appears to keep opening new magnetic flux into interplanetary space with no obvious way for this flux to close back off again. This state of affairs, without some previously unknown method for closing the open interplanetary magnetic field (IMF), leads to an ever growing amount of magnetic flux in interplanetary space: the magnetic flux catastrophe. Recently, considerable progress has been made in understanding why this catastrophic state is not the observed state of the heliosphere. This brief article paints the newly emerging picture of the opening and closing of the IMF and how these processes may account for the observed variation in the amount of magnetic flux in interplanetary space over the solar cycle.
\end{abstract}

\section{Introduction}

Figure 1 displays a composite picture of the Sun's atmosphere or corona from 30 June 1973. The outer portion was taken in ordinary white light from the ground during a solar eclipse. The superposed soft X-ray image of the denser, near-Sun corona was taken from Skylab on the same day. The structure evident in the images is a consequence of the solar magnetic field that permeates the corona. Because of the very high electrical conductivity of the coronal plasma, electrons and ions are bound to specific field lines such that more and less populated lines are mapped out as density structures in these images. Coronal holes are low density regions where the coronal fields open out into interplanetary space; a large coronal hole is displayed as the dark region near the top of Figure 1. In contrast, bright loop-like structures indicate closed field regions on the Sun that contain high density plasma. The centers of helmet streamers (closed field loops overlaid by nearly 
radial high density streamer structures), such as those seen extending outwards to the left and right sides in Figure 1, map out to large current sheets in interplanetary space while the solar wind flow from streamers is slower and higher density than it is from coronal holes [e.g., Borrini et al., 1981]. Coronagraph and soft X-ray observations over the past several decades have shown that the solar corona is highly dynamic and variable with open and closed regions evolving over time scales as short as minutes and as long as the 22 year solar cycle.

Because the interplanetary magnetic field (IMF) is "frozen" into the solar wind plasma by its high electrical conductivity, the concept of spaghetti-like "magnetic field lines" provide a useful paradigm for understanding the magnetic field configuration in interplanetary space. Since the magnetic field is divergence free $(\nabla \cdot \mathbf{B}=0)$, all field lines must be closed. That is, field lines must connect back to themselves 1) back at the Sun, 2) somewhere far out in the heliosphere, or 3) locally in interplanetary space. Figure 2 utilizes a "flat heliosphere" representation to portray the only four fundamentally distinct ways that magnetic field lines in interplanetary space can close. Panel A displays "open" field lines that connect between the Sun and the outer heliosphere. A magnetic tongue geometry, where the magnetic field closes to the Sun at both ends is shown in panel B. Panel C displays a fully disconnected magnetic bottle or plasmoid which closes locally on itself in interplanetary space. Finally, panel D shows disconnected U-shaped field lines extend to the outer heliosphere at both ends. The solar wind flows outward (from bottom to top in Figure 2), so that the field lines in panel B naturally evolve into open field lines (panel A) while field lines in panels $\mathrm{C}$ and $\mathrm{D}$ simply propagate out through the heliosphere and are lost.

Over the past several years, considerable progress has been made in understanding the ways in which open and closed magnetic field regions evolve from the solar corona out through interplanetary space. This paper examines this progress in understanding in four interrelated areas: 1) the opening of new magnetic flux from the Sun; 2) the "flux catastrophe" expected for unbridled opening of flux; 3 ) a newly appreciated method for closing off and returning flux to the Sun; and 4) how a balance between the opening and closing of flux might be maintained.

\section{Opening Magnetic Flux}


Coronal mass ejections (CMEs) [e.g., Gosling et al., 1974; Hundhausen, 1993] are transient eruptions of coronal material and imbedded magnetic field loops which expand outward into interplanetary space. While none were occurring during the time of the image shown in Figure 1, CMEs are often detected in sequences of coronagraph images as bright loops rising up and expanding out of the coronagraph field of view (see, for example, Gosling et al. [1974]). Long lasting, bright radial structures called "legs" are often observed along the flanks of a CME; legs typically persist for many hours after the release of a CME. Since CMEs originate from regions of closed coronal magnetic fields that were not previously participating in solar wind expansion [Gosling et al., 1976], legs have commonly been interpreted as evidence for continued magnetic connection of CMEs back to the Sun (see, however, Kahler and Hundhausen [1992]), CMEs thus open new magnetic flux into interplanetary space.

Solar wind electron distributions are characterized by two components: a thermal population and a suprathermal "halo" population. Halo electrons usually have a beamed component directed along the local magnetic field; these beamed electrons have very long interaction scale lengths and can be treated essentially as test particles tracing out magnetic field topologies. A unidirectional suprathermal electron beam directed outward from the Sun along the IMF indicates field lines that are tied back to a hot $\left(\sim 10^{6} \mathrm{~K}\right)$ corona at one end but not at the other ( $\mathrm{A}$ in Figure 2 ).

In interplanetary space CMEs have been identified by a number of plasma and field signatures (summarized by Gosling [1990]). Of all of these signatures, counterstreaming suprathermal (halo) electrons seem to provide the most unambiguous indication of CMEs in the interplanetary medium [Gosling, 1990; Gosling et al., 1992]. The reason is that just as a unidirectional beam directed outward along the IMF indicates a single connection back to a hot coronal source, counterstreaming can best be interpreted as connection to the Sun in both directions along the IMF ( $B$ in Figure 2). Alternately, if magnetic reconnection pinches off a magnetic tongue to form a plasmoid, ( $\mathrm{C}$ in Figure 2), it is possible that suprathermal electrons already beaming both directions along the field could continue to circulate on such a structure for a long time, owing to their long interaction lengths. Phillips et al. [1992] find that asymmetries between the beams in counterstreaming electron events and variations in the field orientation across CMEs indicate that CMEs maintain at least some magnetic connection back to the corona. In addition, they 
documented the first examples of distributions which displays two broad counterstreaming beams with an additional narrow beam superposed in the direction running most nearly outward from the Sun along the IMF. These distributions provide additional evidence for continued magnetic connection back to the Sun [Phillips et al., 1992].

Roughly one third of all CMEs in interplanetary space exhibit the smooth field rotations characteristic of magnetic clouds or interplanetary flux ropes [Gosiing 1990; 1993]. Figure 3 schematically shows how reconnection between the lower parts of adjacent magnetic arches which are sheared with respect to each other will form a flux rope-type topology and underlying magnetic loops. Of course this process would not be expected to occur only between a single pair of field lines (as shown), but rather across a broad region, producing a long (and potentially magnetically complicated) flux rope-type CME, free to expand into interplanetary space, and an arcade of underlying coronal loops which remain tied to the Sun.

Soft X-ray observations of the lower corona (as in Figure 1) indicate that roughly one third of all CMEs leave underlying arcades [Sheeley et al., 1983]; this fraction is approximately the same as the fraction of all CMEs that have flux rope-like properties in interplanetary space [Gosling, 1993; Gosling et al., 1994]. One particularly clear case study was recently shown by Gosling et al. [1994] in which post CME loop formation observed with the Yohkoh soft X-ray imager was correlated with the subsequent passage of a flux-rope type CME past Ulysses at 4.6 AU. Motions of magnetic footprints down in the turbulent photosphere will naturally lead to shearing of the fields and can also bring the feet closer together increasing the likelihood of reconnection. In such a complicated configuration it is hard to imagine how all of the appropriate field lines could just happen to be jositioned such that reconnection would form a fully detached plasmoid. Rather, it seems far more likely that some amount of magnetic connection is maintained for all CMEs.

\section{The Magnetic Flux Catastrophe}

In the absence of some mitigating processes, the amount of new magnetic flux opened up by the outward expansion of CMEs would grow without bound. This "flux catastrophe" was first pointed out by Gosling [1975]. The amount of open magnetic flux in interplanetary space has been examined most recently using an improved proxy, the "total flux integral" which removes the 
effects of variations in the solar wind speed in determining the amount of magnetic flux crossing 1 $\mathrm{AU}$ [McComas et al., 1992a]. If we assume that all counterstreaming electron events represent simply connected magnetic tongues ( $B$ in Figure 2), then for CME rates characteristic of solar maximum, the amount of flux crossing $1 \mathrm{AU}$ would double over $\sim 9$ months in the absence of any mitigating processes. If CMEs remain magnetically attached but are flux ropes, where field lines can loop around many times in interplanetary space before connecting back to the Sun, then the rate of flux build-up would be smaller. However, it must be stressed that if CMEs retain any attachment to the Sun whatsoever, the flux catastrophe will ultimately occur in the absence of some other process to close off previously open fields.

The average magnitude of the IMF displays $~ 50 \%$ variation over solar cycle 21 [Slavin et al., 1986] while the total flux integral displays $\sim 60 \%$ variation [McComas et al., 1992a,b]; both show basically the same modulation over solar cycle 21 with maxima shortly after solar maximum and minima shortly after solar minimum [McComas et al., 1992a,b; McComas, 1994]. Both this variation over the solar and the fact that the flux catastrophe does not occur, indicates that in addition to CMEs opening new, previously closed field regions from the corona into interplanetary space, there must be some process for closing off previously open field regions and returning magnetic flux to the Sun.

\section{Closing of Flux}

Beyond the point where the solar wind outflow becomes faster than the Alfven speed (typically within a few tens solar radii) the solar wind and frozen-in magnetic field can never contract back to the Sun. In addition, while low altitude arcades are often observed below expanding CMEs, coronagraph observations of the high altitude portions of the corona virtually never show evidence of magnetic structures contracting back toward the Sun. As a consequence, the only reasonable method of reducing the amount of magnetic flux open to interplanetary space seems to be via reconnection between oppositely directed, previously open field lines [McComas et al., 1989, 1991; McComas, 1994]. The top left inset in Figure 4 schematically displays such a sequence of events. Compression of the oppositely directed magnetic field regions across a helmet streamer ultimately leads to reconnection between previously open field lines. The topological effect is to 1) 
create closed field loops that can return to the Sun, and 2) release disconnected $U$-shaped field structures ( $D$ in Figure 2 ) into interplanetary space. An example of such a coronal disconnection event was shown by McComas et al. [1991] using SMM coronagraph images from I June 1989. In that sequence of events a large coronal streamer appears to disconnect creating the appearance of bright loops at lower altitudes and a large, disconnected U-shaped structure above it. Subsequent images show the U-shaped structure accelerating outward from the corona, leaving behind an arcade of bright loops indicative of the newly returned magnetic flux.

The remainder of Figure 4 shows evidence for a possible coronal disconnection as far back as the 16 April 1893 solar eclipse. This figure, adapted from Cliver et al. [1989] by McComas [1994], shows sketches (data in 1893) made in sequence from Chile, Brazil, and Senegal, indicating the outward motion of a large U-shaped structure. While originally interpreted as a comet, Cliver et al. [1989] reinterpreted this sequence as evidence of a fully detached CME (e.g., a plasmoid). While this interpretation might be correct, the sketches do not display any leading edge of a CME. Rather, the observations suggest that a coronal disconnection event occurred.

A statistical study of three months of SMM coronagraph observations [Hundhausen, 1993] was conducted to assess how common coronal disconnection events are [McComas et al., 1992c]. The first three months of 1988, in which an initial SMM survey [St. Cyr and Burkepile, 1990] had found no ubvious disconnections, was resurveyed specifically to look for such events. Of the 53 transient events during this interval, $6(11 \%)$ showed some evidence of disconnection in more than one frame and $13(23 \%)$ showed a single frame with an outward "U" or "V" structure. While these latter structures may not be significant in light of the fact that there are ambiguities in interpreting images from optically thin media, McComas et al. [1992c] concluded that magnetic disconnection events on previously open field lines (above helmet streamers) may be far more common than previously appreciated.

Impulsive low energy ( $<10 \mathrm{keV})$ solar electron events called "high coronal flares" [Cliver and Kahler, 1991] provide another independent line of evidence for magnetic reconnection above helmet streamers. These electrons are inferred to have been accelerated in the corona at heights above the solar surface $>0.5$ solar radii [Potter et al. 1980]. Reconnection above helmet streamers is the most likely location for such high altitude reconnection [Cliver and Kahler, 1991]. Lin [1985] found that such events occur roughly once every three days which suggests that the 
formation of detached coronal magnetic structures may be a relatively common feature of the solar corona.

As discussed above, energetic electrons streaming along the local IMF provide good indications of the magnetic topology in interplanetary space. Since the normal unidirectional heat flux indicates simple magnetic connection to the Sun along open field lines and counterstreaming halo electron events indicate closed magnetic structures, intervals devoid of these suprathermal electrons and the electron heat flux they carry would be logically interpreted as magnetic structures that are disconnected from the Sun and open to the outer heliosphere at both ends ( $D$ in Figure 2) [McComas et al., 1989]. This is true because the hot suprathermal electrons run outward along the field far faster than the disconnected structure moves outward with the bulk solar wind.

Intervals devoid of any suprathermal electron beam, coined "heat flux dropouts" or HFDs, have been found using data from the ISEE-3 solar wind electron experiment [McComas et al., 1989]. While HFDs were identified purely on the basis of the electron distributions, they correlate extremely well with field reversals of the interplanetary magnetic field (current sheet crossings) in general, and crossings of the heliospheric current sheet (sector boundary crossings), in particular [McComas et al., 1989; 1992d]. This association is naturally explained if HFDs arise from reconnection between oppositely directed fields above helmet streamers, at the base of the heliospheric current sheet.

In an independent analysis of HFDs, Lin and Kahler [1992] examined the electron distributions at higher energies (2-8.5 keV); higher energies particles are superior tracers of magnetic topologies compared to $0.1-1 \mathrm{keV}$ halo electrons, whenever they are present. These authors found that of the 25 events on the original HFD list [McComas et al., 1989], nine exhibited strong unidirectional streaming at these higher energies, indicating that the field lines were probably still connected to the Sun. A weak unidirectional streaming was found for 13 of 25 of the identified HFDs, leaving in doubt the connectivity of these events. Finally, two of the cases exhibited clear dropouts even at these higher energies, indicating that at least these events were probably real disconnections.

In addition to the topological explanation for HFDs, enhanced coulomb collisions have been suggested as another possible explanation for HFDs [McComas et al., 1989; Lin and Kahler, 1992]. While scattering may account for some HFDs identified with the lower energy electrons. Lin and Kahler [1992] showed that it cannot account for all of the HFD observations. Finally, the 
possibility of reconnection out in interplanetary space, rather than down in the corona, was examined as another possible source of HFDs [McComas et al., 1994]. These authors showed a case study where reconnection ahead of a faster moving CME driver appears to have converted normal open field lines into a pair of structures: a tongue and a disconnected U-shaped structure, indicated by an HFD. It is worth noting, however, that this sort of reconnection in interplanetary space can not in any way affect the balance of opening and closing flux. HFDs appear not to be unique indicators of coronal disconnection events and the consequent return of magnetic flux to the Sun since both reconnection out in interplanetary space and other processes (possibly enhanced collisions) can also create HFD signatures .

The only suggested method that I am aware of for reducing the amount of open magnetic flux in interplanetary space is through reconnection between oppositely directed open field lines, inside of the distance where the solar wind becomes super-Alfvenic, most likely at the base of the heliospheric current sheet above helmet streamers. This process returns previously open magnetic flux to the Sun as closed arches and can also account for the observations of 1) coronal disconnection events, 2) high coronal flares, and 3) some HFDs.

\section{Balance and Evolution of the IMF}

For the opening and closing of magnetic fields from the Sun to maintain some sort of equilibrium, neither growing nor shrinking without bound, there must be some interaction or feedback between these two processes. One likely interaction is through the transverse magnetic pressure in the solar corona [McComas et al., 1989; 1991; McComas, 1994]. The expansion of newly opened field regions must enhance the transverse magnetic pressure which will compress the open field lines, including those above helmet streamers, elsewhere around the Sun. Ultimately, when enough pressure has built up, reconnection may be initiated which will reduce the amount of open flux, and hence the magnetic pressure. In this way, the opening and closing of flux could maintain a sort of rough equilibrium.

Direct evidence for at least part of this process is provided by the 27 June 1988 coronal disconnection event. In this case, an impulsive compression, indicated by the deflection of the streamers in the corona, preceded, and appeared to initiate the coronal disconnection event 
[McComas et al., 1991]. Another line of supporting evidence is provided by numerical simulations [Linker et al., 1992]. These authors used a 2-D MHD simulation of the corona to examine the effect of increasing the overall magnetic pressure around an equilibrium helmet streamer. They found that increased pressure lead to reconnection of the previously stable streamer configuration which continued until sufficient flux had been removed to again achieve equilibrium.

The opening and closing processes described here could also explain the evolution of magnetic structures back in the corona. Coronal structures such as coronal holes, streamers, and loops, appear, disappear, and evolve on a wide range of times scales, from minutes to years. The global coronal structure also changes from simple and dipolar at solar minimum to very complicated at solar maximum. Both of these sorts of reconfigurations seem to be easier to explain by opening and closing processes than by motions of magneric footprints across huge distances on the Sun.

Acknowledgements Many valuable discussions with and comments on this manuscript by J.T. Gosling are gratefully acknowledged. This work was carried out under the auspices of the United States Department of Energy.

\section{References}

Borrini, G., J.T. Gosling, S.J. Bame, W.C. Feldman, and J.M. Wilcox, Solar wind helium and hydrogen structure near the heliospheric current sheet: A signal of coronal streamers at $1 \mathrm{AU}$, J. Geophys. Res., 86, 4565, 1981.

Cliver, E.V., Was the eclipse comet of 1893 a disconnected coronal mass ejection?, Sol. Phys., 122, 319, 1989.

Cliver, E. and S. Kahler, High coronal flares and impulsive acceleration of solar energetic particles, Astrophys. J., 366, L91-L94, 1991.

Eddy, J.A., The new Sun: The solar results from Skylab, ed. R. Ise, NASA SP-402, Washington D.C., 1979.

Gosling, J.T., Large-scale inhomogeneities in the solar wind of solar origin, Rev. of Geophys. and Space Phys., 13, 1053, 1975.

Gosling, J.T., Coronal mass ejections and magnetic flux ropes in interplanetary space. Physics of 
Magnetic Flux Ropes, ed. C.T. Russell, E.R. Priest, and L.C. Lee, Geophys. Mono. 58, AGU, 343, 1990.

Gosling, J.T., The: solar flare myth, J. Geophys. Res., 98, 18937, 199_.

Gosling, J.T., E. Hildner, R.M. MacQueen, R.H. Munro, A.I. Poland, and C.L. Ross, Magnetic ejections from the Sun: a view from Skylab, J. Geophys. Res., 79, 4581, 1974.

Gosling, J.T., E. Hildner, R.M. MacQueen, R.H. Munro, A.I. Poland, and C.L. Ross, The speeds of coronal mass ejection events, Sol. Phys., 48, 389, 1976.

Gosling, J.T., S.J. Bame, D.J. McComas, J.L. Phillips, B.E. Goldstein, and M. Neugebauer, The speeds of coronal mass ejections in the solar wind at mid heliographic latitudes: Ulysses, in press in Geophys. Res. Lett., 1994.

Hundhausen, A.J., The size and locations of coronal mass ejections: SMM observations from 1980 and 1984-1989, J. Geophys. Res., 98, 13177, 1993.

Kahler, S.W. and A.J. Hundhausen, The magnetic topology of solar coronal structures following mass ejections, J. Geophys. Res., 97, 1619-1631, 1992.

Lin, R.P., Energetic solar electrons in the interplanetary medium, Solar Phys., 100, 537, 1985.

Lin, R.P. and S.W. Kahler, Interplanetary magnetic field connection to the Sun during electron heat flux dropouts in the solar wind, J. Geophys. Res., 97, 8203-8209, 1992.

McComas, D.J., Evolution of the interplanetary magnetic field, in press in AGU monographSolar System Plasma Physics: Resolution of Processes in Space and Time, ed. J. Burch and H. Waite, 1994.

McComas D.J., J.T. Gosling, J.L. Phillips, S.J. Bame, J.G. Luhmann, and E.J. Smith, Electron heat flux dropouts in the solar wind: evidence for interplanetary magnetic field reconnection?, J. Geophys. Res., 94, 6907, 1989.

McComas, D.J., J.L. Phillips, A.J. Hundhausen, and J.T. Burkepile, Observations of disconnection of open coronal magnetic structures, Geophys. Res. Lett., 18, 73, 1991.

McComas, D.J., J.T. Gosling, and J.L. Phillips, Interplanetary magnetic flux: Measurement and balance, J. Geophys. Res., 97, 171, 1992a.

McComas, D.J., J.T. Gosling, and J.L. Phillips, Regulation of the Interplanetary Magnetic Field, Solar Wind 7, ed. E. Marsch and R. Schwenn, Pergamon Press, Oxford, 643-646, $1992 \mathrm{~b}$.

McComas, D.J., J.L. Phillips, A.J. Hundhausen, and J.T. Burkepile, Disconnection of Open 
Coronal Magnetic Structures, Solar Wind 7, ed. E. Marsch and R. Schwenn, Pergamon Press, Oxford, 225-228, 1992c.

McComas, D.J. and J.L. Phillips, The Extension of Solar Magnetic Fields into Interplanetary Space, Proc. of the first SOLTIP Symposium, Vol. 1, edited by S. Fischer and M. Vandas, Czechoslovak Academy of Sciences, Prague, 180-191, 1992d.

McComas, D.J., J.T. Gosling, C.M. Hammond, M.B. Moldwin, J.L. Phillips, and R.J. Forsyth, Magnetic reconnection ahead of a coronal mass ejection, in press in Geophys. Res. Lett., 1994.

Phillips, J.L., J.T. Gosling, D.J. McComas, S.J. Bame, and W.C. Feldman, Quantitative Analysis of Bidirectional Electron Fluxes Within Coronal Mass Ejections at $1 \mathrm{AU}$, Solar Wind 7, ed. E. Marsch and R. Schwenn, Pergamon Press, Oxford, 651-656, 1992.

Potter, D.W., R.P. Lin, and K.A. Anderson, Impulsive 2-10 keV solar electron events not associated with flares, Astrophys. J., 236, L97, 1980.

St. Cyr, O.C. and J.T. Burkepile, A catalog of mass ejections observed by the Solar Maximum Mission coronagraph, NCAR Technical Note, NCAR/TN-352+STR, 1990.

Sheeley, N.R., R.A. Howard, M.J. Koomen, and D.J. Michels, Association between coronal mass ejection events and soft X-ray events, Astrophys. J., 272, 349, 1983.

Slavin, J.A., G. Jungman, and E.J. Smith, The interplanetary magnetic field during solar cycle 21: ISEE-3/ICE Observations, Geophys. Res. Lett., 13, 513, 1986. 


\section{Figure Captions}

Figure 1. Composite picture of the solar corona taken with a white light coronagraph (outer portion) and a soft X-ray imager (inıer portion) on 30 June 1973. Images taken from Eddy [1979].

Figure 2. Schematic diagram showing normal open magnetic fields (A), a magnetic tongue connected to the Sun at both ends (B), a bottle or plasmoid magnetically closed on itself (C), and a coronal disconnection in which the field is open to the outer heliosphere at both ends (D). From McComas et al. [1994].

Figure 3. Schematic diagram of how sheared magnetic loops at the Sun can form a flux-rope topology CME and closed arches beneath it. From Gosling [1993].

Figure 4. Sequence of events for open magnetic field lines to reconnect above a helmet streamer returning flux to the Sun as closed field arches and releasing a disconnected U-shaped structure (top left inset). The map shows the locations of the drawings (three lower insets) of a possible coronal disconnection observed during the 16 April 1893 solar eclipse. Adapted from Cliver et al. [1989] by McComas [1994]. 


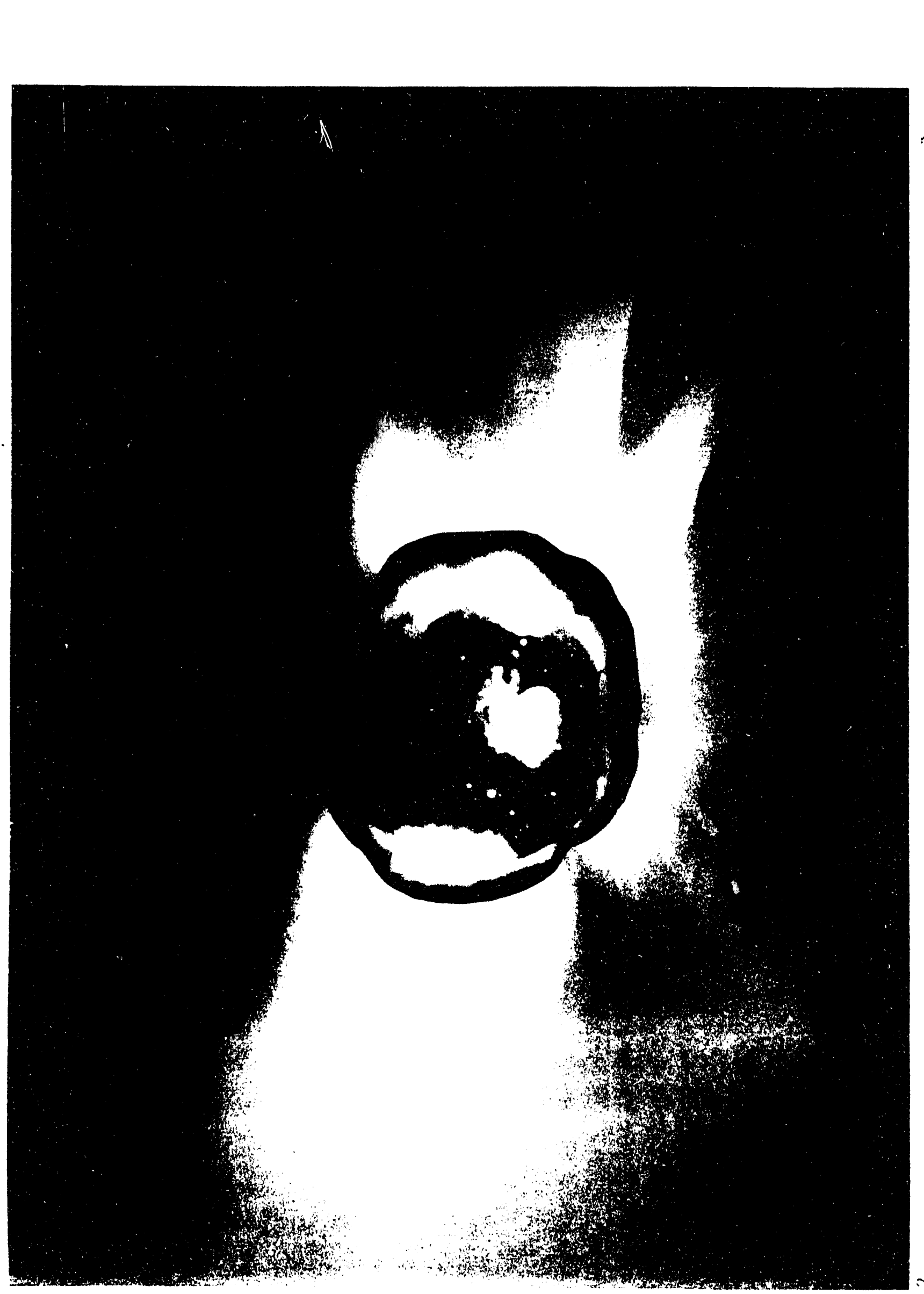




\section{Outer Heliosphere}

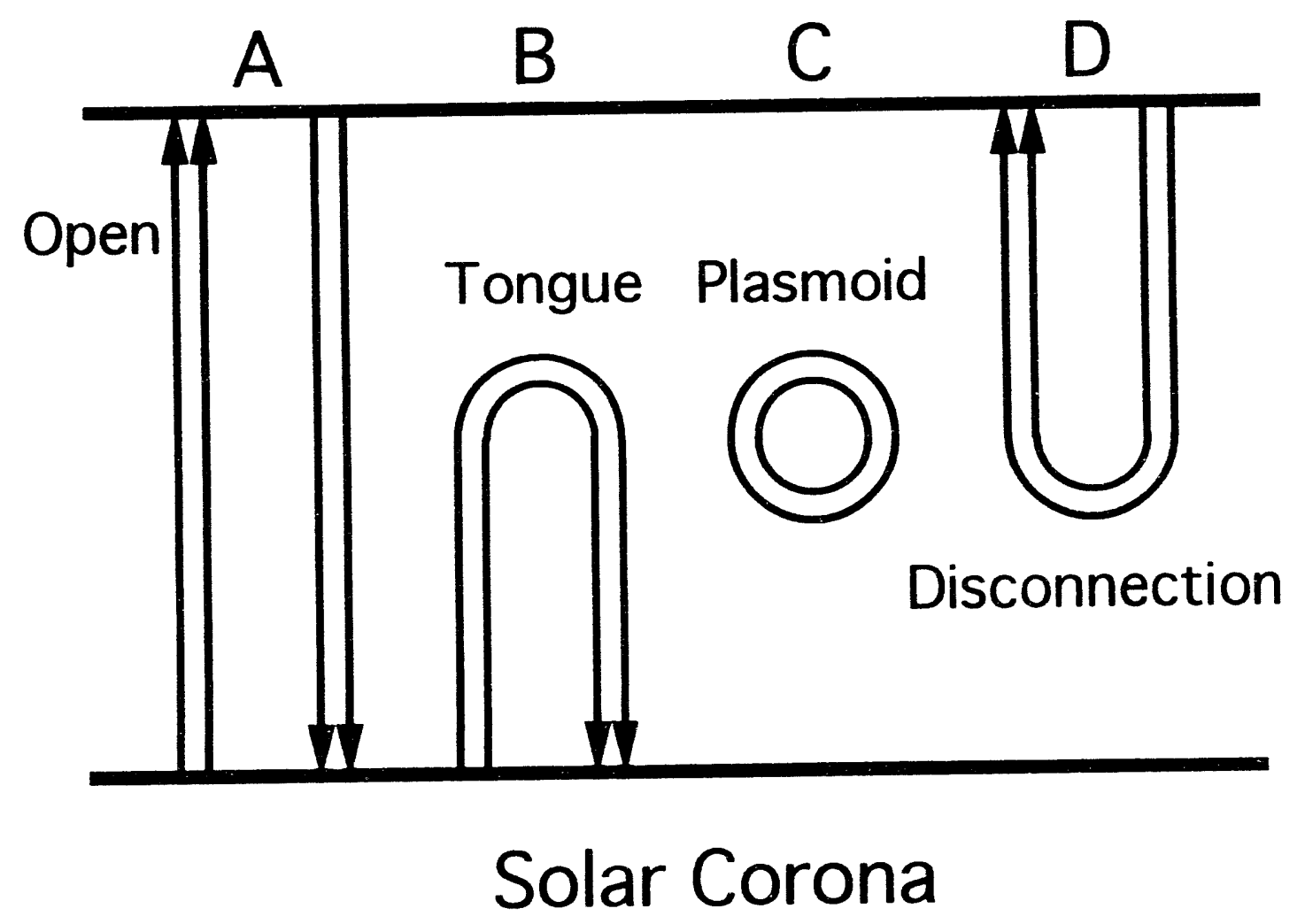

Fig. 2 


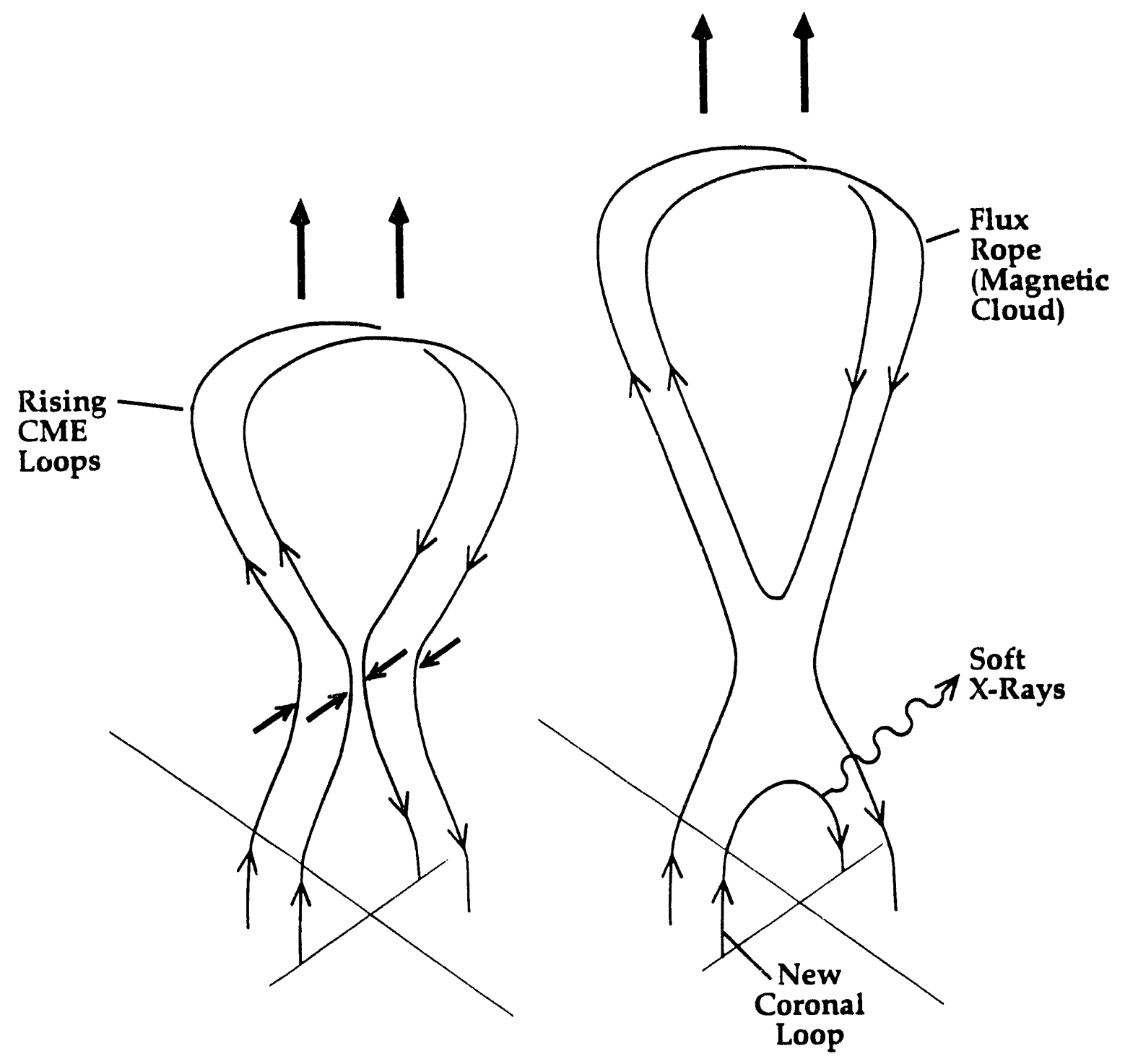

Fig. 3 


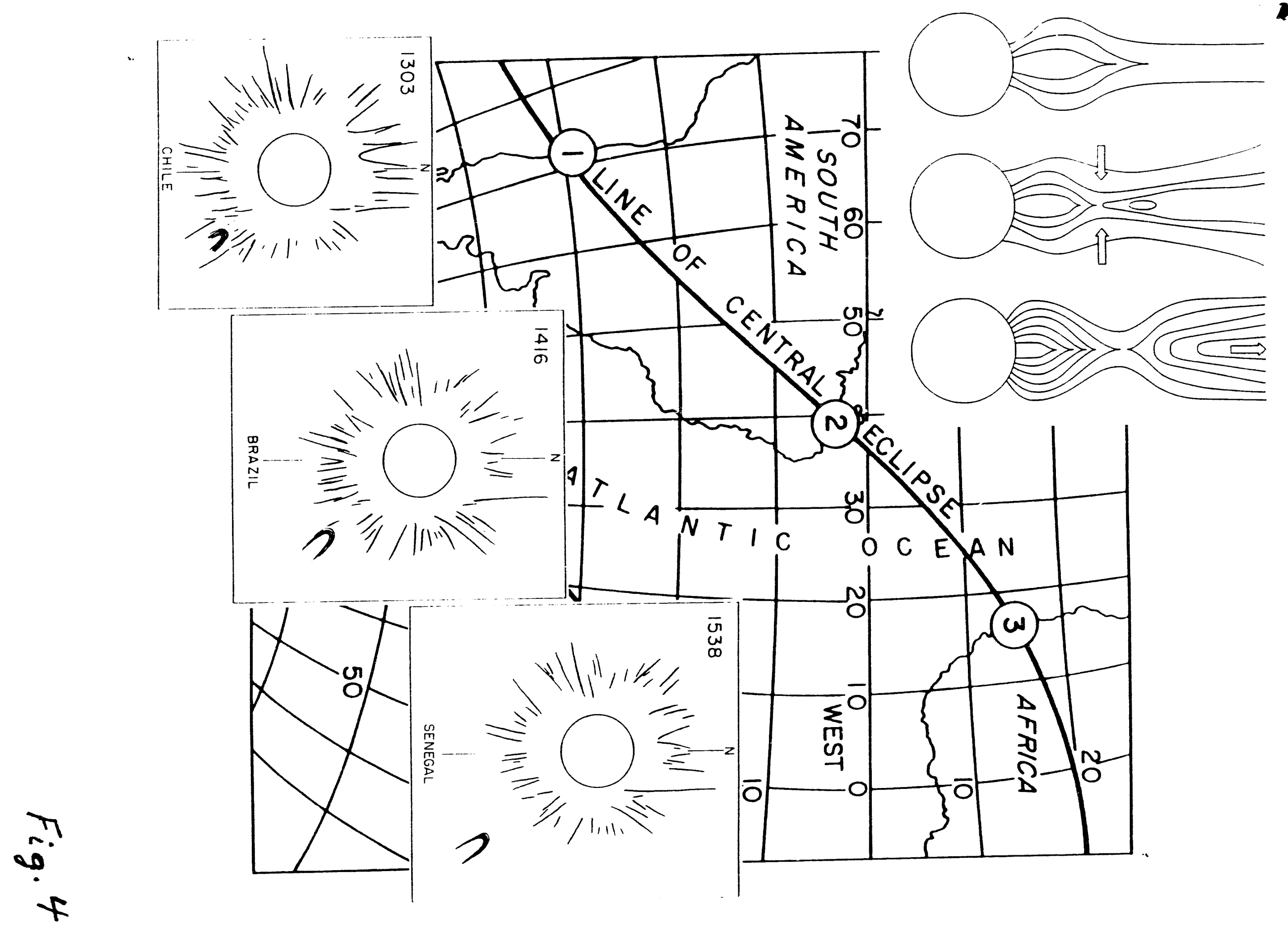



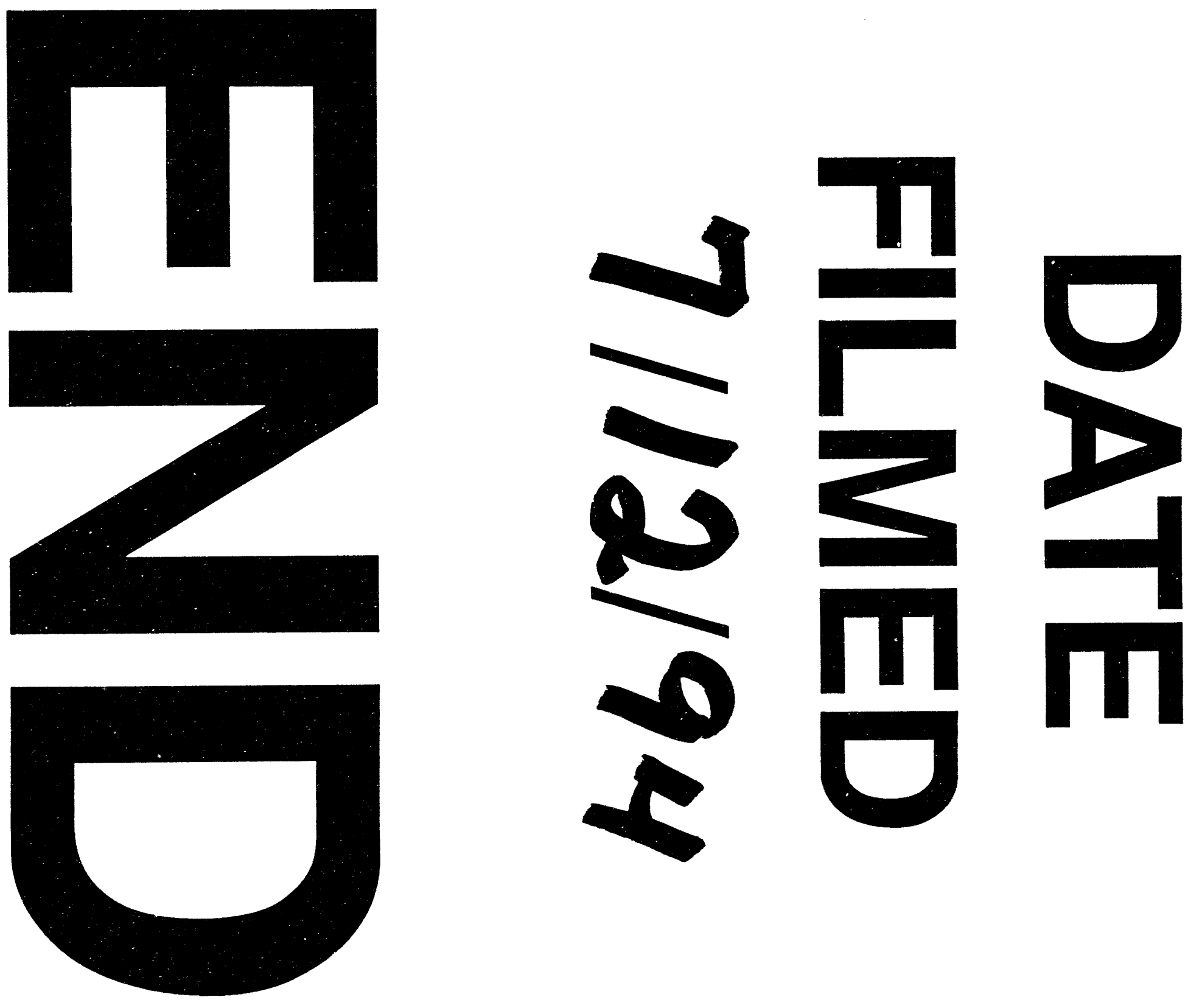

1 
